\title{
Utilization of endobronchial electrocautery for removal of an impacted airway foreign body
}

\author{
Vijay Noel Nongpiur ${ }^{1}$, Saurabh Mittal², Karan Madan, ${ }^{2}$ Anant Mohan², Vijay Hadda ${ }^{2}$ \\ ${ }^{1}$ Department of Tuberculosis and Respiratory Diseases, North Eastern Indira Gandhi Regional Institute of Health and \\ Medical Sciences, Shillong; ${ }^{2}$ Department of Pulmonary, Critical Care and Sleep Medicine, All India Institute of \\ Medical Sciences, New Delhi, India
}

Dear Editor,

Aspiration of a foreign body into the tracheobronchial tree can occur at any age, though it occurs more commonly in children. In small children, the foreign body commonly gets lodged in the central airways (trachea and mainstem bronchi); hence the presentation is usually sudden onset of cough and dyspnea or even lifethreatening respiratory failure in an otherwise healthy child. A witnessed aspiration or a history of aspiration can usually be elicited. In adolescents and adults, however, non-asphyxiating airway foreign bodies are more common, where the foreign body gets lodged in the more distal airways. Acute presentations are less common,

Correspondence: Saurabh Mittal, Department of Pulmonary, Critical Care and Sleep Medicine, All India Institute of Medical Sciences, New Delhi, India.

Tel. +91-11-26593488, +91-9968859414.

E-mail: Saurabh_kgmu@yahoo.co.in

Key words: Bronchoscopy; foreign body; electrocautery.

Funding; None.

Conflict of interest: The authors declare no conflict of interest.

Availability of data and materials: All data underlying the findings are fully available.

Ethics approval and consent to participate: No ethical committee approval was required for this case report by the Department, because this article does not contain any studies with human participants or animals. Informed consent was obtained from the patient included in this study.

Consent for publication: The patient gave his written consent to use his personal data for the publication of this case report and any accompanying images.

Received for publication: 16 May 2020.

Accepted for publication: 26 June 2020.

${ }^{\circ}$ Copyright: the Author(s), 2020

Licensee PAGEPress, Italy

Monaldi Archives for Chest Disease 2020; 90:1383

doi: 10.4081/monaldi.2020.1383

This article is distributed under the terms of the Creative Commons Attribution Noncommercial License (by-nc 4.0) which permits any noncommercial use, distribution, and reproduction in any medium, provided the original author(s) and source are credited. and often the diagnosis is initially overlooked. Such undiagnosed foreign body aspirations lead to symptoms such as recurrent cough, expectoration, and wheezing, which often gets treated as asthma or pneumonia. Delays in diagnosis can lead to complications of long-standing airway foreign bodies such as impaction, erosion of airway wall, lung collapse, and bronchiectasis [1]. Endobronchial removal is the treatment of choice in airway foreign bodies. In this regard, rigid bronchoscopy has many advantages over flexible bronchoscopy, especially in chronic, impacted foreign bodies. Herein, we present a case of an impacted plastic whistle that was successfully removed with the help of electrosurgical cautery through a rigid bronchoscope.

A 17-year-old boy had presented to the outpatient department with a cough for the last seven years. The cough was predominantly dry, but in the last two months, there had been an increase in his cough along with mucoid expectoration. He also gave a history of streaky hemoptysis on and off in these two months. He had no other significant past, personal, or family history. On further questioning, he gave a history of possible aspiration of a whistle seven years back; however, no evaluation or intervention was done for the same. On examination, vitals were stable. Respiratory system examination revealed decreased intensity of breath sounds in the right lung base along with coarse crackles. He was evaluated in an outside hospital, and a chest radiograph and computed tomography of the thorax demonstrated collapse of the right lower and middle lobe along with bronchiectasis (Figure 1). A flexible bronchoscopy demonstrated an impacted foreign body in the right intermediate bronchus. Attempts to remove the foreign body using flexible bronchoscopy had failed, as the intermediate bronchus proximal to the foreign body had stenosis. He was referred to our center for surgical removal. Because of the impacted foreign body, a decision to proceed with rigid bronchoscopic removal was taken. Under gen-

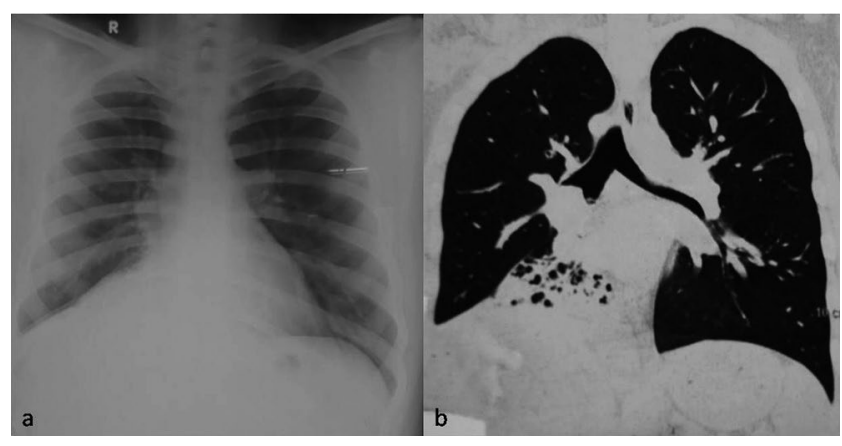

Figure 1. a) Chest radiograph demonstrating right middle and lower lobe collapse. b) CT thorax demonstrating bronchiectasis in right lower lobe. 


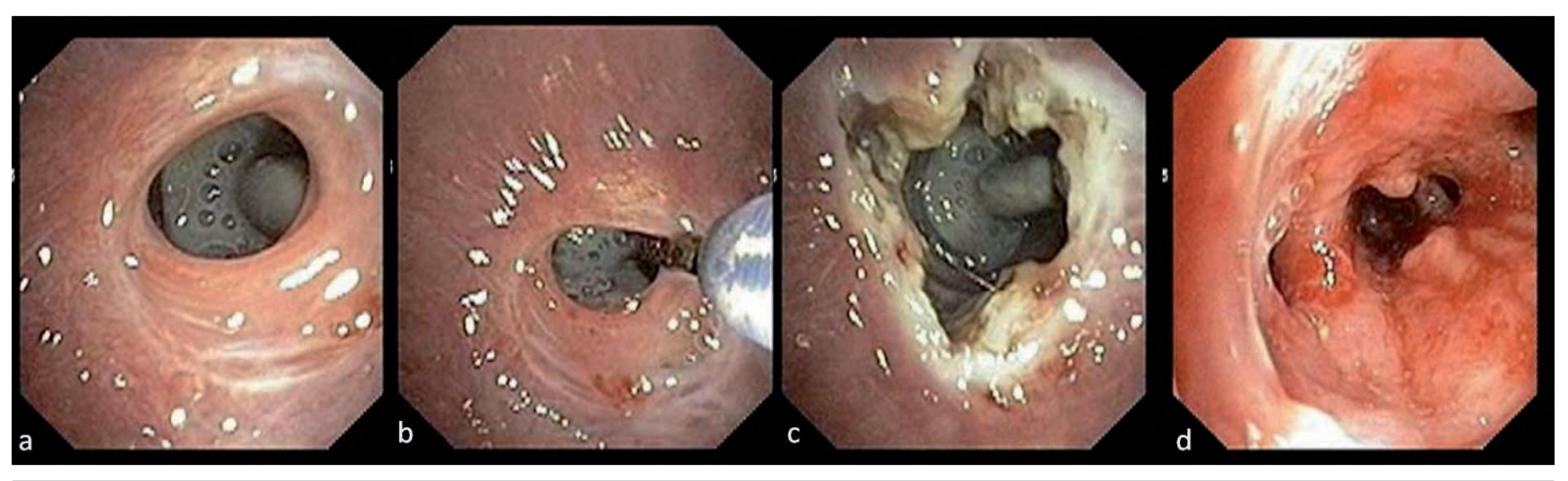

Figure 2. a) Web-like stenosis of right intermediate bronchus. b) Electrocautery knife being used for radial incisions. c) Adequate airway lumen was achieved with multiple incisions. d) Post foreign body removal mild mucosal irregularity was noted.

eral anesthesia, he was intubated with a size 12 rigid tracheobronchoscope. Web-like stenosis of the right intermediate bronchus was seen (Figure 2a), and an impacted foreign body could be seen distal to it. Subsequently, radial incisions were made over the web using an electrocautery knife (Figure 2b). Once airway lumen was sufficient (Figure 2c), the foreign body (plastic whistle, Figure 3a) removal was done using non-optical rigid bronchoscopy forceps. Subsequently, the distal segments were examined with flexible bronchoscopy (Figure 2d), and tracheobronchial toileting was done. He was discharged from the hospital the next day with advice to take oral antibiotics for seven days. The chest radiograph performed after one month demonstrated clearance (Figure $3 \mathrm{~b}$ ) and a check bronchoscopy demonstrated mild stenosis of intermediate bronchus, and distal segments were clear.

Bronchial stenosis following foreign body aspiration is an uncommon occurrence. It is usually reported following aspiration of iron tablets leading to the so-called Iron Pill Aspiration Syndrome, wherein bronchial stenosis occurs due to the caustic

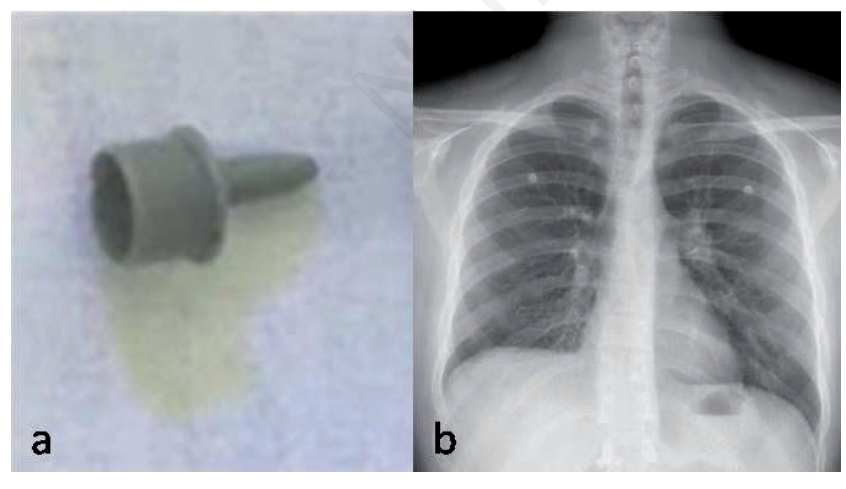

Figure 3. a) The plastic whistle retrieved with rigid bronchoscopy. b) Chest radiograph after one month demonstrating cleared right lower lobe. nature of the metal within the mucosa causing inflammation, and stenosis [2]. Unusually, other foreign bodies (a plastic whistle in this case) can also cause stenosis due to chronic mechanical irritation and subsequent inflammation and fibrosis.

In our case, we used endobronchial electrocautery through a rigid bronchoscope to assist the foreign body removal, thus avoiding surgical intervention. Electrocautery is commonly used for both malignant as well as benign central airway obstructions such as granulation tissues, papillomas, airway stenosis, and in performing a hot biopsy from a highly vascular lesion [3]. In our case, the site of bronchial stenosis was proximal to the location of the foreign body. The use of radial electrocautery incisions expanded the stenosed segment and allowed easy retrieval of the foreign body using non-optical forceps. The other instrument which could have been tried was a controlled radial expansion (CRE) balloon, but because of distal airway non-visualization, it was not deemed appropriate. This approach can be useful in patients with longstanding impacted airway foreign body, keeping a back up of thoracic surgery team for any inadvertent event.

To conclude, a rigid bronchoscope, with its vast array of available instruments and modalities, remains the instrument of choice for foreign body removal. Innovative use of available instruments may be needed for to achieve a successful removal.

\section{References}

1. Aggarwal SK, Sinha SK, Ratan SK, et al. Complications of long-standing foreign body in the airway and their outcomes after endoscopic management: an experience of 20 cases. J Laparoendosc Adv Surg Tech A 2015;25:81-7.

2. Lee P, Culver DA, Farver C, Mehta AC. Syndrome of iron pill aspiration. Chest. 2002;121:1355-7.

3. Sachdeva A, Pickering EM, Lee HJ. From electrocautery, balloon dilatation, neodymium-doped:yttrium-aluminumgarnet (Nd:YAG) laser to argon plasma coagulation and cryotherapy. J Thorac Dis 2015;7:S363-79. 